\title{
ARTICLE OPEN Calculation and interpretation of classical turning surfaces in solids
}

\author{
Aaron D. Kaplan $\mathbb{D}^{1}$, Stewart J. Clark $\mathbb{D}^{2 凶}$, Kieron Burke $\mathbb{i}^{3 凶}$ and John P. Perdew ${ }^{4 凶}$
}

Classical turning surfaces of Kohn-Sham potentials separate classically allowed regions (CARs) from classically forbidden regions (CFRs). They are useful for understanding many chemical properties of molecules but need not exist in solids, where the density never decays to zero. At equilibrium geometries, we find that CFRs are absent in perfect metals, rare in covalent semiconductors at equilibrium, but common in ionic and molecular crystals. In all materials, CFRs appear or grow as the internuclear distances are uniformly expanded. They can also appear at a monovacancy in a metal. Calculations with several approximate density functionals and codes confirm these behaviors. A classical picture of conduction suggests that CARs should be connected in metals, and disconnected in wide-gap insulators, and is confirmed in the limits of extreme compression and expansion. Surprisingly, many semiconductors have no CFR at equilibrium, a key finding for density functional construction. Nonetheless, a strong correlation with insulating behavior can still be inferred. Moreover, equilibrium bond lengths for all cases can be estimated from the bond type and the sum of the classical turning radii of the free atoms or ions.

npj Computational Materials (2021)7:25; https://doi.org/10.1038/s41524-020-00479-0

\section{INTRODUCTION}

Modern Kohn-Sham (KS) density functional theory (DFT) ${ }^{1}$ calculations produce a KS potential, $v_{s}(\mathbf{r})$, which, while not a physical observable, has proven useful in providing physical and chemical insight. Recently, the classical turning surfaces at the energy of the highest occupied orbital of atoms and ions have provided considerable insight into the nature of chemical bonding ${ }^{2}$. These turning surfaces are roughly ellipsoidal for covalent bonds, have seams for ionic bonds, and are bifurcated for van der Waals dimers. But all finite systems have densities that decay to zero far from the nuclei, whereas the interiors of real solids extended over three dimensions do not. Thus, unlike molecules, solids have the possibility of having no classical turning surfaces at all. Here we augment existing codes to calculate classical turning surfaces for a variety of extended solids.

We first find that equilibrium distances between nearestneighbor atoms or atomic ions in solids can be estimated from the sum of the classical turning radii of the corresponding free atoms or atomic ions and the bond type (metallic, covalent, ionic, or van der Waals), much as found earlier for molecules. But of greater interest is the nature of the turning surface itself in solids. Physical intuition suggests that, in the limit of extreme compression, solids become metallic, while, in the less-physical limit of extreme expansion, they become insulators. In the former case, there are no classical turning surfaces, i.e., all space is classically allowed for the most energetic electrons, while in the latter, all atoms (or ions) are isolated spheres from which classical electrons could not escape. Semiclassical reasoning suggests that solids that are entirely classically allowed should be metallic, while a significant volume of forbidden regions should accompany greater inhibition of conduction, i.e., a nonzero gap. Thus we might naively expect semiconductors with covalent bonds to have classical turning surfaces.
We calculated many solids as a function of lattice parameter using the local spin density approximation (LSDA) and the Perdew-Burke-Ernzerhof (PBE) generalized gradient approximation $(\mathrm{GGA})^{3}$, tracking the fraction of volume that is classically forbidden. The results are shown in Fig. 1. The single most important feature is that bulk metals at equilibrium have no classically forbidden regions (CFRs). This is consistent with the original views of Kohn and Sham when developing $\mathrm{DFT}^{1}$, and the subsequent use of gradient expansions starting from slowly varying densities. The next most important feature is surprising: at equilibrium, covalent semiconductors also do not exhibit any CFRs. This has two important consequences. First, this shows that such systems differ fundamentally from their molecular cousins. Covalent molecules do not have forbidden regions between nuclei, but do in other directions. Covalent solids have none.

Second, this is consistent with the general behavior of density functional approximations, such as GGAs versus (global) hybrids and meta-GGAs. The exact exchange-correlation hole in most metals is short-ranged ${ }^{4}$, is adequately described by the LSDA, and more accurately described by GGAs ${ }^{5}$. In some cases (e.g., magnetic properties of transition metals), GGAs better describe metals ${ }^{6,7}$ than meta-GGAs $s^{8,9}$ and hybrids ${ }^{10}$, which typically do better for semiconductors $^{11-13}$ and insulators ${ }^{12,13}$ presumably because metals exhibit perfect screening, whereas semiconductors and insulators do not.

But the heuristic suggested by semiclasscial physics is also not entirely without merit. If we expand the volume of a semiconductor by $40 \%$, we found, in every case but one, the appearance of a CFR. So semiclassical reasoning is sound, but not quite quantitative. The one exception is diamond where, according to our bonding characterization, the atomic densities are strongly overlapped.

These observations are broad strokes, based on the simple solids shown in Fig. 1. A theoretical background immediately

\footnotetext{
Department of Physics, Temple University, Philadelphia, PA 19122, USA. ${ }^{2}$ Centre for Materials Physics, Durham University, Durham DH1 3LE, UK. ${ }^{3}$ Departments of Chemistry and Physics, University of California, Irvine, CA 92697, USA. ${ }^{4}$ Departments of Physics and Chemistry, Temple University, Philadelphia, PA 19122, USA. ${ }^{\circledR}$ email: s.j.clark@durham.ac.uk; kieron@uci.edu; perdew@temple.edu
} 


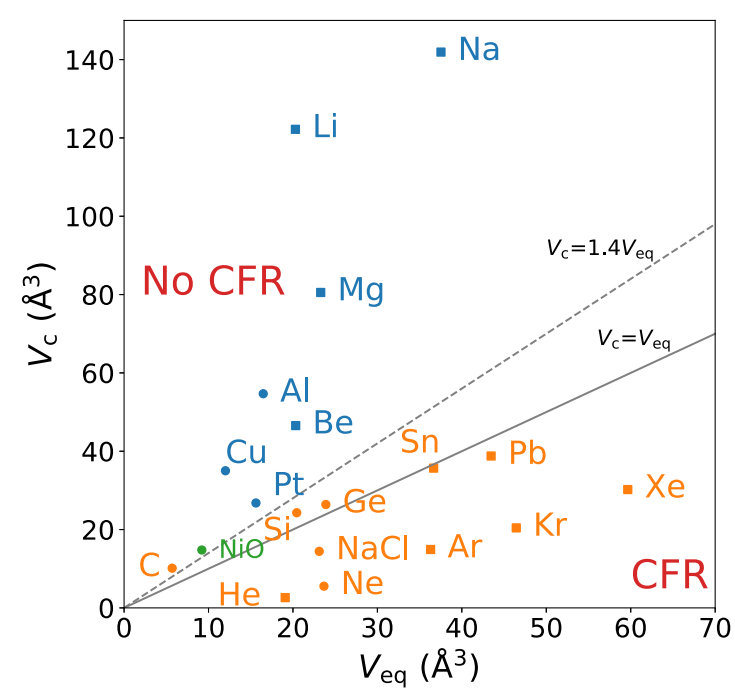

Fig. 1 CFRs at equilibrium for a variety of metals and insulators. Contrasting the amount of strain needed to induce a CFR for various solids at smaller equilibrium volume per atom $V_{\text {eq }}$, for PBE only. For the complete figure, see Supplementary Fig. 4. Solids lying above the gray line $V_{c}=V_{\text {eq }}$ have no CFR at equilibrium, and those below the line have a CFR at equilibrium. The line $V_{c}=1.4 V_{\text {eq }}$ better separates metals and insulators; the only exceptions are diamond and $\mathrm{NiO}$. For clarity of the figure, only the rocksalt antiferromagnetic (AFM) phase of $\mathrm{NiO}$ (green) is shown; other phases are discussed later.

follows this overview. Our analysis begins with a generalization of the chemical bonding analysis to solids. We also examine several other interesting effects, often by studying one specific case. We show that insertion of a defect in Pt creates a CFR. An exploration of the fraction of CFR volume created as a function of lattice expansion reveals strong patterns running down columns of the periodic table. Changes of crystalline phase in $\mathrm{Al}$ as well as magnetic and crystalline phases in $\mathrm{NiO}$ are explored. As a prototype of complexity, $\mathrm{NiO}$ does not fit neatly into the picture of Fig. 1, regardless of phase. The Supplementary Information contains additional figures and data.

The most basic properties of an ordered solid are its conductivity $^{14-16}$ and bonding ${ }^{17}$. The standard quantum mechanical theory of conduction for ordered solids is that of Bloch bands, with insulators having filled bands below finite gaps in the eigenvalue spectrum ${ }^{17}$. The local bonding within a solid is often determined qualitatively from the real-space charge density.

Consider a classical electron of energy $\varepsilon$ moving in a oneelectron effective potential $v_{\text {eff }}(\mathbf{r})$. If $\varepsilon>v_{\text {eff }}(\mathbf{r})$ everywhere, the classical electron will traverse the solid uninhibited, and the solid should be metallic. But, if the only classically allowed regions (CARs) are disjoint regions bound to atoms, the solid should be strongly insulating. Unlike the classical electron, a quantum electron can tunnel into the CFR, permitting small conductivity.

This work analyzes turning surface analogs in the framework of $\mathrm{KS} \mathrm{DFT}^{1}$, an exact-in-principle quantum theory of many electrons. In KS DFT, the exact ground-state density and total energy are given by an auxiliary system of non-interacting electrons subject to an effective scalar potential $v_{s}(\mathbf{r})$. For consistency with previous work $^{2}$, we define the turning surface of the KS potential as the set of points $\mathbf{r}_{\mathrm{c}}$ such that

$v_{\mathrm{s}}\left(\mathbf{r}_{\mathrm{c}}\right)=\varepsilon_{\mathrm{HO}}$,

with $\varepsilon_{\mathrm{HO}}$ the highest occupied orbital energy (or Fermi energy $\varepsilon_{\mathrm{F}}$ ). If $\varepsilon_{\mathrm{HO}}>v_{s}(\mathbf{r})$ everywhere, there is no turning surface. (One could also define a turning surface using the chemical potential $\mu \geq \varepsilon_{\mathrm{HO}}{ }^{18}$, with equality only for metals, but Eq. (1) is used in all previous work.)

Highly accurate approximations of the KS potential ${ }^{19,20}$ are computationally tractable in atoms and molecules, but more difficult in solids. The existing literature on KS turning surfaces considers finite systems exclusively ${ }^{2,21-24}$. Replacing the core regions by a pseudopotential, and explicitly excluding the core regions from the analysis of the potential (as is done here), may countervail the lack of core-region structure in approximate KS potentials. Pronounced structures in the exact KS potential may feature in the low-density interstices of stretched solids, well inside CFRs.

Solids, as opposed to atoms and molecules, offer the possibility of metallic bonds, no classical turning surface (as suggested for metals by the cartoon of ref. ${ }^{25}$ ), a sharp distinction between zerotemperature electrical conduction and insulation, and physical spontaneous symmetry-breaking (as in transition metal monoxides). Analysis of the spin-sublattices requires a further definition of the sublattice-, or spin-, CFRs

$v_{\mathrm{s}, \sigma}(\mathbf{r})=\varepsilon_{\sigma, \mathrm{HO}}, \quad \sigma=\uparrow, \downarrow$.

The presence of a classical turning surface, as at a monovacancy in a metal, is responsible for strong Friedel oscillations of the electron density ${ }^{26}$. Moreover, quantum oscillations (shell structure) in the density are observed in the CAR, while the density decays exponentially within the $\mathrm{CFR}^{26}$.

This work presents calculations of turning surfaces for many simple solids at the LSDA and GGA levels of exchange-correlation approximations. Both usually yield close approximations to more precise KS potentials in molecules (as both KS potential and $\varepsilon_{\mathrm{HO}}$ are typically too shallow by about the same amount). However, the bandgap of the exact KS potential does not match the fundamental or physical gap ${ }^{18}$, and typically underestimates it. The bandgaps of semilocal approximations like LSDA or GGAs are typically close to the exact KS bandgap ${ }^{27-29}$, and thus underestimate the fundamental bandgap. Hybrid functionals and metaGGAs yield larger bandgaps when treated in a generalized KS scheme ${ }^{30}$. To determine the turning surface of a hybrid functional or meta-GGA, one would need to replace ${ }^{20}$ the non-multiplicative potential operator of generalized KS theory with a multiplicative one.

Semilocal approximations to the KS potential may deteriorate under extreme expansive strains. The exact KS potential need not be analytic nor smooth, as seen in the intershell regions of atoms $^{31}$ and stretched molecular bonds ${ }^{18,27,32}$. However, even if a negative hydrostatic pressure could be achieved, extreme expansion of the lattice is unphysical: the work needed to stretch the lattice will eventually exceed the surface formation energy, signaling a transition to isolated clusters.

\section{RESULTS}

\section{Characterizing bonding in solids}

It is often useful to identify the general chemical properties of materials. Crystals are generally classified as metallic (conducting), covalently bonded, ionically bonded, hydrogen bonded, or van der Waals bonded (the latter four being insulating). However, it is difficult to quantifiably distinguish between these classifications in terms of the KS potential or density alone. The design of metaGGAs has led to an "iso-orbital indicator" $a(\mathbf{r})$ that describes the local chemical environment quantitatively ${ }^{9,33}, a(\mathbf{r}) \rightarrow 0$ characterizes one- and two-electron regions, $a(\mathbf{r}) \rightarrow 1$ uniform densities, and $a(\mathbf{r}) \gg 1$ weak bonds.

It was shown in ref. ${ }^{2}$ that the bond type roughly determines the ratio

$\beta_{\mathrm{AB}}=\frac{s_{\mathrm{AB}}}{R_{\mathrm{A}}+R_{\mathrm{B}}}$, 
Table 1. Chemical bonding properties from atomic turning surface radii.

\begin{tabular}{|c|c|c|c|c|c|c|}
\hline Solid & Struc. & $V_{\mathrm{c}} / V_{\text {eq }}$ & $\beta_{\mathrm{AB}}$ & $s_{A B}(\AA)$ & Bond & Bond type \\
\hline C & hex & $<1$ & 0.478 & 1.424 & C-C I.L. & Cov. \\
\hline C & ds & 1.778 & 0.519 & 1.547 & $C-C$ & Cov. \\
\hline $\mathrm{C}^{*}$ & ds & 1.899 & 0.520 & 1.548 & $\mathrm{C}-\mathrm{C}$ & Cov. \\
\hline Al & fcc & 3.318 & 0.549 & 2.857 & Al-Al & Met. \\
\hline $\mathrm{Al}^{*}$ & fcc & 2.503 & 0.550 & 2.858 & Al-Al & Met. \\
\hline $\mathrm{Si}^{*}$ & ds & 1.262 & 0.551 & 2.367 & $\mathrm{Si}-\mathrm{Si}$ & Cov. \\
\hline $\mathrm{Si}$ & ds & 1.188 & 0.551 & 2.368 & $\mathrm{Si}-\mathrm{Si}$ & Cov. \\
\hline $\mathrm{Li}^{*}$ & $\mathrm{bcc}$ & 6.022 & 0.553 & 2.976 & $\mathrm{Li}-\mathrm{Li}$ & Met. \\
\hline Ge & ds & 1.105 & 0.564 & 2.494 & Ge-Ge & Cov. \\
\hline $\mathrm{Ge}^{*}$ & ds & 1.150 & 0.565 & 2.498 & $\mathrm{Ge}-\mathrm{Ge}$ & Cov. \\
\hline $\mathrm{Cu}$ & $f c c$ & 2.916 & 0.574 & 2.570 & $\mathrm{Cu}-\mathrm{Cu}$ & Met. \\
\hline $\mathrm{Cu}^{*}$ & fcc & 3.033 & 0.574 & 2.571 & $\mathrm{Cu}-\mathrm{Cu}$ & Met. \\
\hline $\mathrm{MoS}_{2}$ & $\mathrm{MoS}_{2}$ & $<1$ & 0.587 & 2.413 & Mo-S I.L. & Cov. \\
\hline$S n^{*}$ & ds & 0.973 & 0.590 & 2.877 & $S n-S n$ & Cov. \\
\hline $\mathrm{Na}^{*}$ & bcc & 3.782 & 0.628 & 3.653 & $\mathrm{Na}-\mathrm{Na}$ & Met. \\
\hline $\mathrm{K}^{*}$ & bcc & 3.146 & 0.634 & 4.555 & $\mathrm{~K}-\mathrm{K}$ & Met. \\
\hline $\mathrm{Rb}^{*}$ & bcc & 2.801 & 0.646 & 4.963 & $\mathrm{Rb}-\mathrm{Rb}$ & Met. \\
\hline $\mathrm{Ca}^{*}$ & $\mathrm{fcc}$ & 3.651 & 0.696 & 3.910 & $\mathrm{Ca}-\mathrm{Ca}$ & Met. \\
\hline $\mathrm{Sr}^{*}$ & $\mathrm{fcc}$ & 3.347 & 0.700 & 4.314 & $\mathrm{Sr}-\mathrm{Sr}$ & Met. \\
\hline $\mathrm{Mg}^{*}$ & bcc & 3.459 & 0.715 & 3.116 & $\mathrm{Mg}-\mathrm{Mg}$ & Met. \\
\hline $\mathrm{NiO}$ AFM & $z b$ & 1.115 & 0.754 & 1.945 & $\mathrm{Ni}^{+2}-\mathrm{O}^{-2}$ & Ion. \\
\hline NiO Unp. & rs & 1.642 & 0.806 & 2.080 & $\mathrm{Ni}^{+2}-\mathrm{O}^{-2}$ & Met. \\
\hline $\mathrm{NiO}$ AFM & rs & 1.605 & 0.812 & 2.096 & $\mathrm{Ni}^{+2}-\mathrm{O}^{-2}$ & Ion. \\
\hline $\mathrm{Be}^{*}$ & bcc & 2.290 & 0.832 & 2.978 & $\mathrm{Be}-\mathrm{Be}$ & Met. \\
\hline $\mathrm{NaCl}$ & rs & 0.625 & 1.249 & 2.849 & $\mathrm{Na}^{+1}-\mathrm{Cl}^{-1}$ & lon. \\
\hline $\mathrm{NaCl}^{*}$ & rs & 0.621 & 1.252 & 2.854 & $\mathrm{Na}^{+1}-\mathrm{Cl}^{-1}$ & Ion. \\
\hline$X e^{*}$ & $\mathrm{fcc}$ & 0.507 & 1.282 & 4.386 & Xe-Xe & $\mathrm{vdW}$ \\
\hline $\mathrm{MoS}_{2}$ & $\mathrm{MoS}_{2}$ & $<1$ & 1.361 & 4.574 & S-S O.L. & $v d W$ \\
\hline C & hex & $<1$ & 1.363 & 4.061 & C-C O.L. & $v d W$ \\
\hline $\mathrm{Kr}^{*}$ & fcc & 0.440 & 1.372 & 4.035 & $\mathrm{Kr}-\mathrm{Kr}$ & $\mathrm{vdW}$ \\
\hline $\mathrm{Ar}^{*}$ & $f c c$ & 0.411 & 1.441 & 3.717 & $\mathrm{Ar}-\mathrm{Ar}$ & $\mathrm{vdW}$ \\
\hline $\mathrm{Ne}^{*}$ & fcc & 0.253 & 1.800 & 3.132 & $\mathrm{Ne}-\mathrm{Ne}$ & $\mathrm{vdW}$ \\
\hline $\mathrm{Ne}$ & $\mathrm{fcc}$ & 0.234 & 1.852 & 3.223 & $\mathrm{Ne}-\mathrm{Ne}$ & $v d W$ \\
\hline $\mathrm{He}^{*}$ & $\mathrm{fcc}$ & 0.138 & 2.419 & 3.000 & $\mathrm{He}-\mathrm{He}$ & $v d W$ \\
\hline
\end{tabular}

Classification of bonding in solids using $\mathrm{PBE}$, ordered by increasing $\beta_{\mathrm{AB}}$ of Eq. (3) (roughly, by decreasing bond strength). An asterisk indicates a Castep calculation, otherwise the result is from VASP. $V_{c}$ is the critical volume per atom at which a CFR appears. For those solids where fitting was performed, a numeric value of $V_{\mathrm{c}} / V_{\mathrm{eq}}$ is given; if no fit was performed, the appropriate inequality is given. In general, $V_{\mathrm{c}} / V_{\text {eq }}>1$ indicates that a solid has no CFR at equilibrium, and $V_{\mathrm{c}} / V_{\text {eq }}<1$ indicates that it does. "I.L." corresponds to an in-layer bond, and "O.L." to an out-of-layer bond. $R_{\mathrm{A}}$ and $R_{\mathrm{B}}$ are the turning surface radii of the corresponding atoms or ions as calculated in ref. ${ }^{2}$. For $\mathrm{Na}^{+1} \mathrm{Cl}^{-1}\left(\mathrm{Ni}^{+2} \mathrm{O}^{-2}\right)$, the isolated monovalent (divalent) ionic turning surface radii were used; neutral atomic radii were used otherwise. The antiferromagnetic (AFM) NiO $V_{c} s$ are identical for spinup and spin-down CFRs. For an analogous table using the LSDA, refer to Supplementary Table I.

with $s_{A B}$ the nearest-neighbor separation of ion sites $A$ and $B$ in a solid or molecule and $R_{\mathrm{A}}$ and $R_{\mathrm{B}}$ are their corresponding classical turning surface radii calculated for the isolated species ${ }^{2}$. As seen in Table 1 , the value of $\beta_{A B}$ is correlated with the bond-type of a crystal. Note that, while $R_{\mathrm{A}}$ and $R_{\mathrm{B}}$ are spherical, $s_{\mathrm{AB}}$ is determined by the lattice geometry. $\beta_{\mathrm{AB}} \lesssim 0.8$ generally indicates a solid with dense covalent bonding or metallic bonding, and a lack of a CFR at equilibrium. $0.8 \lesssim \beta_{\mathrm{AB}} \lesssim 1.3$ indicates an ionic crystal, and $1.3 \lesssim$ $\beta_{\mathrm{AB}}$ indicates a van der Waals crystal, with a CFR at equilibrium in both cases. Note that this classification differs from that in ref. ${ }^{2}$ only in the upper bound for covalent bonding, which was 0.7 in their work, and the lower ionic bound, which they took to be 0.9 . Equation (3) might be used to guess an initial geometry for a novel material of given composition.

We note a few outliers: $\mathrm{Sn}$ and $\mathrm{Pb}$ as calculated in the diamond structure, which have $\beta_{A B}<0.8$ and a CFR at equilibrium; and $\mathrm{NiO}$ and $\mathrm{Be}$, which have $0.8<\beta_{\mathrm{AB}}<1.3$, but no CFRs at equilibrium. The ground-state structures of $\mathrm{Sn}$ and $\mathrm{Pb}$ are not diamond cubic, thus the presented lattices are not true equilibrium phases. As shown later, lattice strains also induce CFRs. $\mathrm{NiO}$ and $\mathrm{Be}$ are discussed further in later sections.

Graphite and $\mathrm{MoS}_{2}$ feature strong covalent in-plane bonds and weak out-of plane bonds. The strongly different LSDA and PBE $V_{c} /$ $V_{\text {eq }}$ values demonstrate the limitations of semilocal functionals in describing weak bonds. We have not considered hydrogen bonding-a type of weak bonding reserved for solid state hydrates-as there are numerous phases of ice that would likely exhibit a range of bonding ${ }^{34}$.

Table 1 shows that the turning surface of a solid at its equilibrium geometry reflects its chemical bonding. CFRs arise between neighboring atoms when the atomic CARs are not overlapped $\left(\beta_{A B}>1\right)$, making the local electron density relatively low. Metallic bonds, and dense networks of covalent bonds, lack turning surfaces and cannot be distinguished in Table 1 (although the valence electron densities of covalent solids tend to be much more inhomogeneous than those of simple metals; see Figs. 6-6 and 6-7 of ref. ${ }^{35}$ for the densities of $\mathrm{Si}$ and $\mathrm{Al}$ ). lonic and van der Waals bonds have classical turning surfaces whose shapes could distinguish them, just as for molecules ${ }^{2}$.

\section{Detailed studies}

We define and report $V_{\mathrm{UC}}$ as the volume per atom of the unit cell (UC), which is independent of the choice of UC (primitive, conventional, etc.). Similarly, we define the CFR fractional volume as the portion of the UC volume, which is classically forbidden. The "Methods" section describes how these quantities may be extracted from standard plane-wave DFT codes used here, the Vienna ab initio Simulation Package (VASP) and Castep.

As seen in Tables 1 and 2, the studied defect-free metals lack CFRs at equilibrium. Table 1 and Fig. 2 show that substantial expansive strains are needed to induce CFRs in metals. The fitting method and fit parameters are described in the Supplementary Information. From Tables 1 and 2 and Supplementary Table II, $V_{\mathrm{c}}$ I $V_{\text {eq }} \gtrsim 3$ for metals, except for Pt where it is 1.7.

Note also that the LSDA and PBE curves in Fig. 2 and Supplementary Fig. 6 for $\mathrm{Al}, \mathrm{Cu}$, and $\mathrm{NaCl}$ cross, whereas those for elemental insulators do not. For the elemental insulators, the difference between the LSDA and PBE curves is always of the same sign.

The equilibrium CFRs of insulators, as in Tables 1 and 2, depend upon the degree of insulation and the approximation used. Thus not all covalent solids have CFRs at equilibrium. Figure 3 plots the turning surface in Si at 30\% expansion of the lattice parameter. In the figure, both the CAR and CFR are simultaneously connected. Supplementary Fig. 5 shows how CARs disconnect under strong expansion. Crystalline $\mathrm{NaCl}$, just like its molecular form ${ }^{2}$, also has large PBE and LSDA CFRs. Because $\mathrm{NaCl}$ is a prototypical ionic solid, ionic crystals and more weakly bound crystals will likely exhibit CFRs at equilibrium.

Weakly interacting and van der Waals solids, like graphite and $\mathrm{Ne}$, have PBE CFRs at equilibrium. The small (1\%) PBE CFR volume in graphite (hexagonal C) at its experimental lattice constants reflects the semimetallic nature of this material. The PBE CFR in graphite lies between monolayers, as one might expect for few- 
Table 2. Turning surface properties of solids at equilibrium.

\begin{tabular}{|c|c|c|c|c|c|c|}
\hline Solid (structure) & & $\varepsilon_{\mathrm{HO}}-v_{\mathrm{s}}^{\max }(\mathrm{eV})$ & CFR fraction & $V_{\text {eq }}\left(\AA^{3} /\right.$ atom $)$ & Lattice const(s). ( $\AA$ ) & $\begin{array}{l}\text { Expt. lattice const(s). } \\
(\AA)\end{array}$ \\
\hline \multirow{2}{*}{\multicolumn{2}{|c|}{$\mathrm{Al}(\mathrm{fcc})$}} & 5.75 & $0 \%$ & 16.48 & 4.04 & $4.02^{64}$ \\
\hline & & (5.94) & & $(15.81)$ & (3.98) & \\
\hline \multirow{2}{*}{\multicolumn{2}{|c|}{$\mathrm{Cu}(\mathrm{fcc})$}} & 5.56 & $0 \%$ & 12.01 & 3.63 & $3.59^{64}$ \\
\hline & & $(6.04)$ & & (10.94) & $(3.52)$ & \\
\hline \multirow{2}{*}{\multicolumn{2}{|c|}{ Pt (fcc, bulk) }} & 4.76 & $0 \%$ & 15.61 & 3.97 & $3.91^{65}$ \\
\hline & & $(5.04)$ & & $(14.90)$ & $(3.90)$ & \\
\hline \multirow{4}{*}{\multicolumn{2}{|c|}{ Pt monovacancy (fcc) }} & -1.18 & $10.9 \%$ & 15.39 & 3.95 & 3.91 \\
\hline & & $(-1.00)$ & $(5.2 \%)$ & (14.68) & (3.89) & \\
\hline & & -1.29 & $12.1 \%$ & 15.61 & 3.97 & 3.91 \\
\hline & & $(-1.12)$ & $(6.4 \%)$ & $(14.90)$ & $(3.90)$ & \\
\hline \multicolumn{2}{|l|}{$C(\mathrm{ds})$} & $5.59(6.62)$ & $0 \%$ & $5.70(5.52)$ & $3.57(3.53)$ & $3.55^{64}$ \\
\hline \multicolumn{2}{|l|}{ Si (ds) } & $0.91(1.44)$ & $0 \%$ & $20.44(19.71)$ & $5.47(5.40)$ & $5.42^{64}$ \\
\hline \multicolumn{2}{|l|}{$\mathrm{Ge}(\mathrm{ds})$} & 0.57 & $0 \%$ & 23.89 & 5.76 & $5.64^{64}$ \\
\hline \multirow[t]{2}{*}{ C (hex) } & Relaxed & $-3.06(1.04)$ & $18.5 \%(0 \%)$ & $10.70(8.61)$ & $2.47(2.45)(a), 8.12(6.65)(c)$ & $2.46(a), 6.71(c)^{66,67}$ \\
\hline & Expt. & $-0.28(0.92)$ & $1.0 \%(0 \%)$ & 8.81 & $2.46(a), 6.71(c)$ & $2.46(a), 6.71(c)$ \\
\hline \multirow[t]{2}{*}{$\mathrm{Ne}(\mathrm{fcc})$} & Relaxed & $-14.44(-9.23)$ & $87.1 \%(78.1 \%)$ & $23.67(14.39)$ & $4.56(3.86)$ & $4.46^{36}$ \\
\hline & Expt. & $-14.20(-10.90)$ & $86.3 \%(86.3 \%)$ & 22.24 & 4.46 & 4.46 \\
\hline \multirow[t]{2}{*}{$\mathrm{NaCl}(\mathrm{rs})$} & Relaxed & $-3.38(-2.05)$ & $34.6 \%(17.7 \%)$ & $23.11(20.45)$ & $5.70(5.47)$ & $5.57^{64}$ \\
\hline & Expt. & $-3.06(-2.30)$ & $29.6 \%(22.4 \%)$ & 21.59 & 5.57 & 5.57 \\
\hline \multicolumn{2}{|c|}{$\mathrm{MoS}_{2}\left(\mathrm{P} 6 / \mathrm{mmc}\right.$ or $\left.2 \mathrm{H}_{b}\right)$} & $-4.24(0.01)$ & $22.3 \%(0 \%)$ & $21.41(16.99)$ & $\begin{array}{l}3.18(3.12)(a), 14.62(12.07)(c), 3.12 \\
(3.11)(z)\end{array}$ & $\begin{array}{l}3.16(a), 12.29(c), 3.17 \\
(z)^{62}\end{array}$ \\
\hline \multirow[t]{3}{*}{$\mathrm{NiO}$} & Unp. (rs) & 4.97 & $0 \%$ & 9.00 & 4.16 & $4.17^{50}$ \\
\hline & AFM (rs) & 3.92 & $0 \%(\uparrow), 0 \%(\downarrow)$ & 9.21 & 4.19 & \\
\hline & AFM (zb) & 0.23 & $0 \%(\uparrow), 0 \%(\downarrow)$ & 11.34 & 4.49 & \\
\hline
\end{tabular}

PBE and LSDA (parenthesized when different) values for the classically forbidden regions, equilibrium volume per atom, and lattice constants of select metals and insulators. For the first set of Pt monovacancy results, the cell volume and ion positions were relaxed; for the second set, the volume was fixed to the bulk value, and the ion positions were relaxed. Both sets of calculations used 31 ions in the supercell. For graphite, $\mathrm{Ne}$, and $\mathrm{NaCl}$, two sets of results are shown: the first at a relaxed PBE geometry, and the second at the experimental equilibrium geometry. The percent volume is taken with respect to the unit cell (percent volume per atom). Here "ds" refers to diamond structure, "hex" to simple hexagonal structure (with a four-point basis for graphite), "rs" to rock salt structure, and "zb" to Zincblende structure. The layered structure of $\mathrm{MoS}_{2}$ is itself a prototype for dichalcogenide structure and is often referred to as the "MoS 2 structure," or by its polytype $2 \mathrm{H}_{b}{ }^{62}$, or by its space group P6/mmc ${ }^{63}$. The $a$ and $c$ parameters have the same meaning as in a simple hexagonal lattice, the $z$ parameter (sometimes called 2z) is the spacing between neighboring sulfur layers. No LSDA calculation was performed for the NiO phases nor for Ge. Spinunpolarized (Unp.) and antiferromagnetic (AFM) calculations for $\mathrm{NiO}$ are listed; the AFM rs state is the correct ground state.

layer graphene. The large PBE CFR volume in graphite, nearly $20 \%$ of the equilibrium volume, is due to PBE's underestimation of intermediate-range van der Waals interactions between layers. This fraction is reduced to $1 \%$ when the experimental cell volume is used instead. The LSDA finds no CFR in graphite, likely due to the LSDA's overestimation of weak interactions. This trend also manifests in $\mathrm{MoS}_{2}$ : the LSDA underestimates the equilibrium $c$ lattice parameter, yielding no CFR. PBE vastly overestimates the $c$ parameter, yielding a substantial CFR. Note that the LSDA and PBE are similarly accurate for the intra-layer thickness $z$ (the distance between neighboring layers of sulfur atoms).

Consider instead a monolayer of graphite or $\mathrm{MoS}_{2}$. For these, the bulk equilibrium $a$ and $z$ lattice parameters were used. No CFR exists within the monolayer region of graphene or monolayer $\mathrm{MoS}_{2}$, for both the LSDA and PBE. Thus no in-layer CFR is present in graphene or monolayer $\mathrm{MoS}_{2}$.

Barring diamond $\mathrm{C}$, which has much stronger covalent bonding than the other elements in the Carbon group, all narrow gap insulators studied here have $V_{\mathrm{c}} / V_{\text {eq }} \lesssim 1.4$. As semilocal functionals provide lower bounds to the bandgap-and in the case of $\mathrm{Ge}$, find zero bandgap - a semilocal calculation could recognize narrow gap insulators by two properties: a nonzero bandgap and/or the presence of a CFR when the UC is expanded within $40 \%$ of the equilibrium volume. As most metals have $V_{\mathrm{c}} / V_{\text {eq }} \gtrsim 1.7$ ( $\mathrm{Pt}$ is the edge case with $\left.V_{\mathrm{c}} / V_{\text {eq }}=1.71\right)$, the expanded criterion could also identify spin-unpolarized and antiferromagnetic (AFM) $\mathrm{NiO}$ as non-metallic.

The classical radius of the free $\mathrm{Ne}$ atom is $0.87 \AA$, in both PBE (Ospadov et al., manuscript in preparation), and with a more accurate KS potential ${ }^{2}$, with a volume of $2.76 \AA^{3}$. The experimental lattice constant is $4.464 \AA^{36}$, corresponding to a cell volume per atom of $22.24 \AA^{3}$. The CFR predicted by ref. ${ }^{2}$ is then $(22.24-$ $2.76) / 22.24 \approx 88 \%$ of the total cell volume, agreeing with the values in Table 2. An Ne atom in solid $\mathrm{Ne}$ at the equilibrium lattice constant is very similar to a free $\mathrm{Ne}$ atom.

We can compress the Ne lattice until the CFR vanishes, as seen in Fig. 2. The Ne CFR is predicted to vanish at $0.62 a$ for PBE. One might expect the bandgap to shrink as the CFR collapses, but the opposite is true. For the smallest lattice constant calculated here $(2.85 \AA)$, the band gap is roughly $18.57 \mathrm{eV}$, compared to a gap of about $11.51 \mathrm{eV}(11.45 \mathrm{eV})$ at the PBE equilibrium (experimental) lattice constant, consistent with previous work that used PBE to study phases of $\mathrm{Ne}$ under pressure ${ }^{37}$. Intuition suggests that the $\mathrm{Ne}$ CFR should not be fully suppressed before the classical turning surfaces between adjacent atoms just touch, at a nearest-neighbor separation of $2(0.87)=1.74 \AA$, using the result from ref. ${ }^{2}$. This is substantially smaller than the nearest-neighbor spacing in crystalline $\mathrm{Ne}$ for which the PBE CFR is wiped out, $2.81 / \sqrt{2} \approx 2.00 \AA$. 


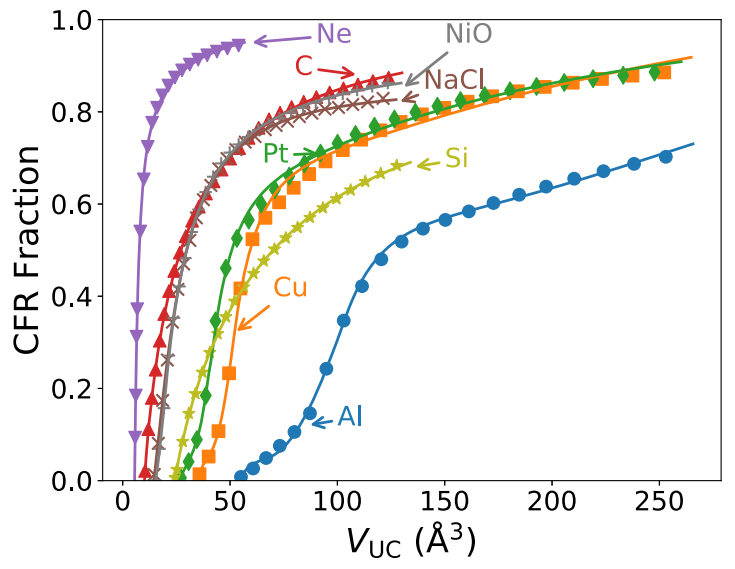

Fig. 2 The role of strain in shaping CFRs. Emergence of PBE CFRs and their volume fractions as functions of the volume per atom in $\mathrm{Al}$ (blue closed circles), $\mathrm{Cu}$ (yellow squares), Pt (green diamonds), C (red point-up triangles), $\mathrm{Ne}$ (purple point-down triangles), $\mathrm{NaCl}$ (brown $\mathrm{Xs}$ ), $\mathrm{Si}$ (olive stars), and $\mathrm{NiO}$ (gray plus signs) as a function of the unit cell volume. All lines are fits given in Supplementary Table II. As Al, $\mathrm{Cu}, \mathrm{C}$, and Si have no CFR at their relaxed lattice parameters, each lattice must be stretched to introduce a CFR. Conversely, $\mathrm{Ne}$ and $\mathrm{NaCl}$ must be compressed to eliminate their CFRs; for completeness, the full $\mathrm{NaCl}$ curve is presented here. The curve for spin-unpolarized $\mathrm{NiO}$ is almost identical to that of $\mathrm{NaCl}$ (see also Supplementary Fig. 3). The LSDA curves are very similar, see Supplementary Fig. 6. For PBE curves as calculated in Castep, see Supplementary Fig. 7.

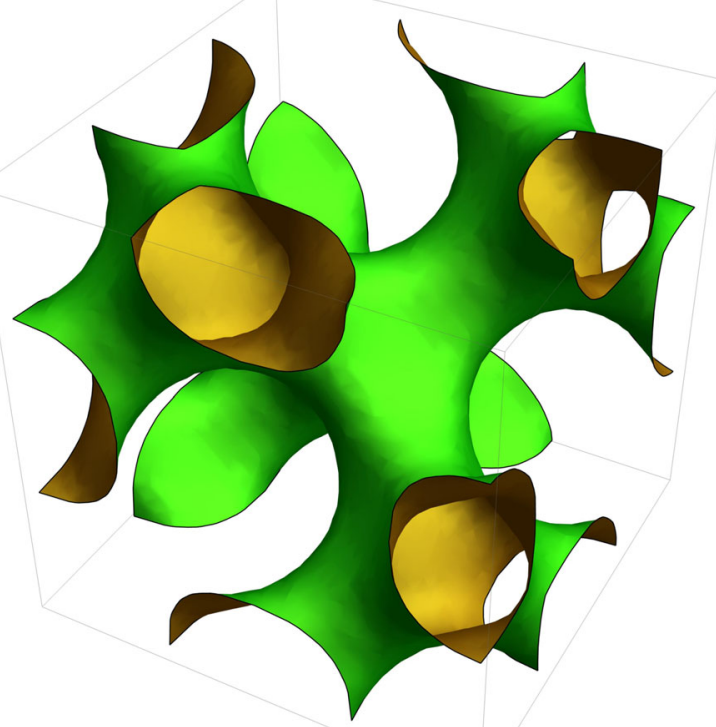

Fig. 3 The KS turning surface of $\mathrm{Si}$ at mild expansion. The surface shows the CAR (outside, green) and CFR (inside, yellow) for silicon at $1.30 a$, with $a$ the equilibrium cubic lattice constant. Both regions are simultaneously fully connected.

Thus, unexpectedly, the critical lattice constant in Ne makes the nearest-neighbor distance noticeably greater than twice the turning radius of the free atom.

As the lattice is compressed, two competing effects determine the bandgap: the bands widen, reducing the gap; and the center of the conduction band is shifted upwards with respect to the center of the valence band, widening the gap. (For an example, see the silicon density of states (DOS) at equilibrium and at a mild expansion in Supplementary Fig. 1.) This leads to a nontrivial (non-

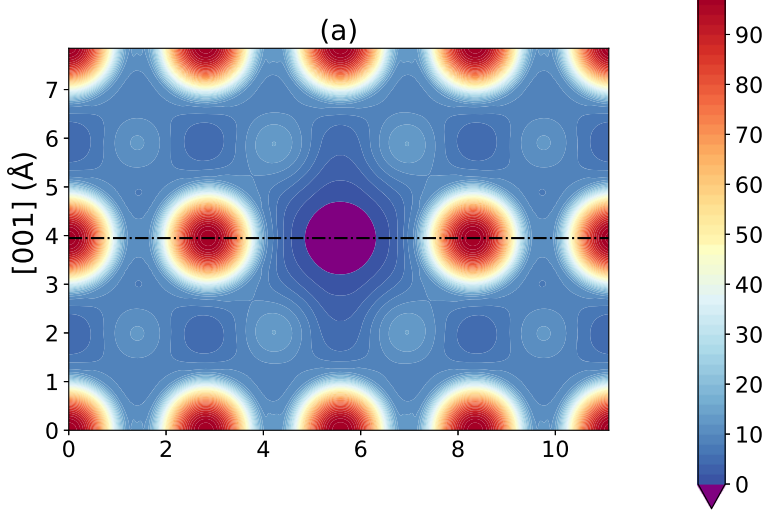

(b)

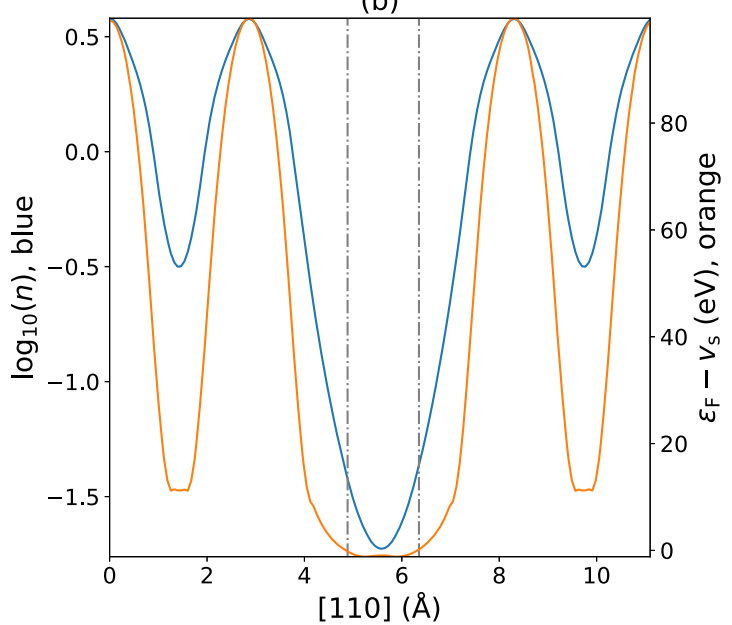

Fig. 4 Creation of a CFR by a monovacancy defect in Pt. a A contour plot of $\varepsilon_{\mathrm{HO}}-v_{s}(\mathbf{r})$ as calculated with PBE along the $(1,-1,0)$ plane (conventional cubic indices) in the Pt monovacancy supercell. The CFR (purple) surrounds the defect, supporting the conjecture that the formation of a defect is accompanied by the formation of an internal curved surface. Regions within the PAW pseudopotential core radii are only included here to make the image clearer. For an analogous figure in $\mathrm{Si}$, refer to Supplementary Fig. 2. b Logarithm of the density $\log _{10}[n(\mathbf{r})]$ (blue) and $\varepsilon_{\mathrm{HO}}-v_{\mathrm{s}}(\mathbf{r})$ (orange) plotted along the black line in $\mathbf{a}$. The density decays exponentially as it crosses the border of the CFR, marked by gray vertical lines.

monotonic) dependence of the gap upon the lattice parameter. Note also that certain crystalline phases of $\mathrm{Na}$ have been experimentally observed to transition to an insulating state under strong compression, consistent with predictions made by $\mathrm{PBE}^{38}$.

\section{The defect case}

A Pt supercell with a monovacancy defect harbors a small CFR, and as seen in Fig. 4, the CFR encapsulates the center of the vacancy perfectly. Relaxation of the supercell volume was performed by two ways: direct minimization of the stress tensor, and allowing ion positions to change within a fixed supercell volume.

The vacancy defect formation energy can be recast as the energy needed to create a curved surface within a solid ${ }^{39}$. The localization of the CFR to the vacancy region is a clear manifestation of this. Carling et al. ${ }^{40}$ found that the LSDA is more accurate than GGAs for the Al monovacancy formation energy, in line with earlier results ${ }^{41}$ for the jellium surface energy. They also found a very low electron density near the center of the vacancy, and large Friedel oscillations around it, consistent with a CFR near the center. Large voids and exterior surfaces would also give rise 
Table 3. Emergent periodic trends.

\begin{tabular}{llcl}
\hline Solid (structure) & $V_{\mathrm{c}} / V_{\text {at }}$ & Solid (structure) & $V_{\mathrm{c}} / V_{\mathrm{at}}$ \\
\hline $\mathrm{Li}(\mathrm{bcc})$ & 1.50 & $\mathrm{Be}(\mathrm{bcc})$ & 1.94 \\
$\mathrm{Na} \mathrm{(bcc)}$ & 1.37 & $\mathrm{Mg}(\mathrm{bcc})$ & 1.86 \\
$\mathrm{~K}(\mathrm{bcc})$ & 1.18 & $\mathrm{Ca}(\mathrm{fcc})$ & 1.66 \\
$\mathrm{Rb}(\mathrm{bcc})$ & 1.11 & $\mathrm{Sr}(\mathrm{fcc})$ & 1.55 \\
& & $\mathrm{He}(\mathrm{fcc})$ & 2.63 \\
$\mathrm{C}(\mathrm{ds})$ & 0.78 & $\mathrm{Ne}(\mathrm{fcc})$ & 1.99 \\
$\mathrm{Si}(\mathrm{ds})$ & 0.62 & $\mathrm{Ar}(\mathrm{fcc})$ & 1.66 \\
Ge (ds) & 0.61 & $\mathrm{Kr}(\mathrm{fcc})$ & 1.54 \\
Sn (ds) & 0.59 & $\mathrm{Xe} \mathrm{(fcc)}$ & 1.44 \\
\hline
\end{tabular}

Comparison of the fitted critical volumes per atom $V_{c}$ for the emergence of a CFR and the CAR volume of isolated atoms for select elemental solids. $V_{\text {at }}=4 \pi r_{\mathrm{TS}}^{3} / 3$, where $r_{\mathrm{TS}}$ is the turning surface radius of the corresponding neutral atom as reported in ref. ${ }^{2}$. Each group of the periodic table, separated by solid lines, seems to approach a column-dependent limit for $V_{\mathrm{c}} / V_{\text {at }}$ as the nuclear charge $Z$ grows large.

to extensive CFRs in any material. Their definition of the monovacancy volume used the liquid drop model of jellium ${ }^{39}$ and will generally yield larger volumes than the corresponding CFR volumes.

\section{The periodic trends}

Here we consider elemental solids beyond those emphasized in Table 2. They are members of Groups 1 (alkali metals), 2 (alkaline earth metals), 14 (Group IV or Carbon group), and 18 (noble gases). The parameters of the fit functions can be found in Supplementary Tables IV-VII and the full strain curves in Supplementary Figs. $8-11$. As seen in Fig. 1 , the line $V_{\mathrm{c}} / V_{\text {eq }} \approx 1.4$ generally distinguishes metals and insulators.

Clear trends in the strain curves of elemental solids emerge as one goes down a column of the periodic table in Table 3 (see also Supplementary Tables IV-VII). In Fig. 5a, we plot the strain curves as a function of $V_{U C} / V_{c}$, for elemental insulators. The noble gases all fall on one line, except for the lightest, $\mathrm{He}$, while the Carbon group elements fall on another, except for the lightest, $C$. Each group has a unique, characteristic curve.

The alkali and alkaline-earth metals show similar but more complex behavior, as shown in Fig. 5b. The green line is for the heavier alkalis, the orange line is for the alkaline-earths. The lighter two alkalis, $\mathrm{Li}$ and $\mathrm{Na}$, are shown in blue and share a shape distinct from the later alkalis. They follow the alkaline-earth curve closely, except for a dip around $1.4 \mathrm{~V}_{\mathrm{c}}$. Moreover, $\mathrm{Mg}$ (in gray) is the odd one out of the alkaline-earths, rather than Be. For small strains, Mg behaves like all other alkaline-earths but, when greatly expanded, behaves more like an alkali.

Naturally, within a column of the periodic table, the critical CFR volume $V_{c}$ increases with atomic number, as shown in the Supplementary Information. Defining the volume of a free atom as $V_{\mathrm{at}}=4 \pi r_{\mathrm{TS}}^{3} / 3$, with $r_{\mathrm{TS}}$ the radius of the atom's classical turning surface ${ }^{2}$, then the ratios $V_{\mathrm{c}} / V_{\text {at }}$ are order 1 and seem to approach a column-dependent large- $Z$ limit, with $Z$ the nuclear charge (see Table 3 and Supplementary Tables IV-VII). The first ionization energies of the atoms exhibit similar behavior ${ }^{42}$.

\section{Phases of Aluminum}

As the volume of the UC changes, the equilibrium crystal phase may no longer be the ground-state phase, leading to a structural phase transition. Semilocal functionals can find states of broken symmetry of lower energy than symmetry-preserving states ${ }^{43}$. In addition to varying crystalline phases, we may also account for
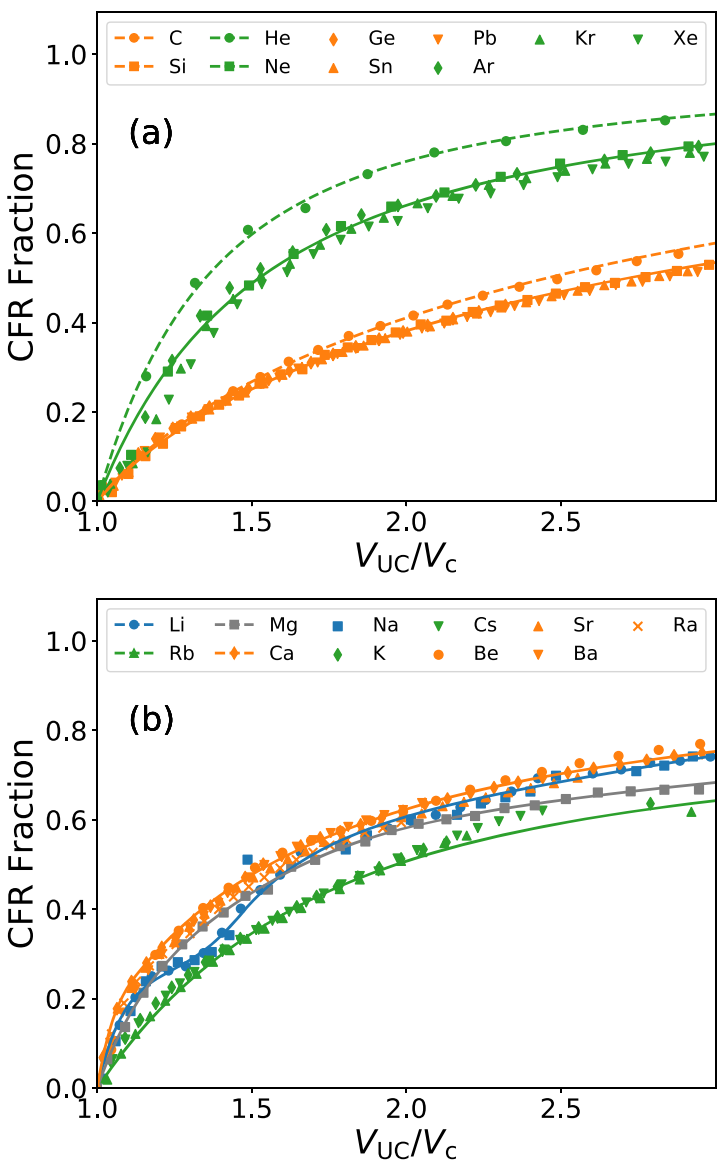

Fig. 5 Periodic trends in CFR evolution under strain. Trends among groups of elements emerge when plotting the CFR fraction against the dimensionless $V_{U C} / V_{c}$, where $V_{U C}$ is the volume per atom and $V_{c}$ is the fitted critical volume per atom. All data here were calculated with PBE in Castep. $\mathbf{a}$ is for the insulators and $\mathbf{b}$ for the metals.

whether a spin-unpolarized phase or a symmetry-broken phase is the true ground state.

For simplicity, we have selected a few cubic phases, facecentered cubic (fcc), body-centered cubic (bcc), simple cubic (sc), and diamond structure cubic (ds), in spin-unpolarized Al to probe possible phase transitions. Using the stabilized jellium equation of state (SJEOS) ${ }^{44}$ to fit the energy per formula unit as a function of the UC volume, PBE predicts an energy crossover for fcc and ds Al at a cubic lattice parameter $a=5.36 \AA$, as can be seen in Fig. 6 . By following the CFR curve of lowest energy in Fig. 6 , the change in inflection in the fcc Al curve is removed. A bandgap in the DOS does not accompany the crystalline phase transition. From the SJEOS parameters, we find the equilibrium volume per atom to be $16.62 \AA^{3}$ for the fcc phase (-3.74 eV/formula unit) and $28.27 \AA^{3}$ for the ds phase $(-2.93 \mathrm{eV} /$ formula unit). The critical transition pressure from the fcc to the ds phase is $-0.070 \mathrm{eV} / \AA^{3}$ $(-11.25 \mathrm{GPa})$, as constructed from the common tangent of the equations of state. A negative hydrostatic pressure is not experimentally realizable.

\section{Nickel monoxide}

Transition metal monoxides are often poorly described by semilocal density functionals, particularly LSDA and GGAs. The spin-unpolarized state respects the full symmetry of the Hamiltonian, but calculations with approximate functionals often find a broken-symmetry AFM state of lower energy. Spin-symmetry breaking in a density-functional calculation is well known to 

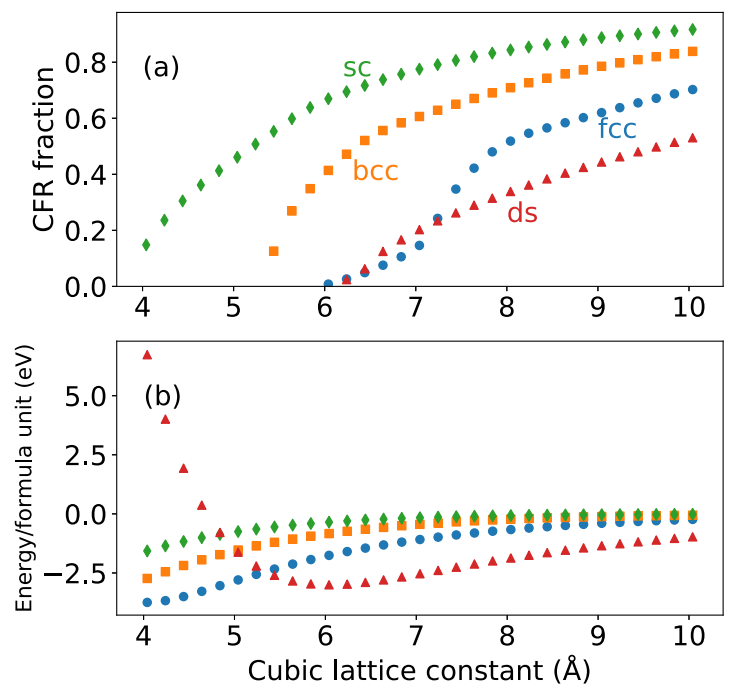

Fig. 6 Competing crystalline phases in Al and their CFRs. a CFR evolution for different spin-unpolarized cubic crystalline phases of $\mathrm{Al}$, as calculated with PBE in VASP. $\mathbf{b}$ The $\mathrm{fcc}$ and ds phases become degenerate near $a=5.36 \AA$ The fcc, bcc, and sc phases become nearly degenerate in energy as a grows large. Note that absolute energies do not have physical meaning in a pseudopotential calculation, only differences in energies. The fcc, bcc and sc phases were taken to contain one formula unit in the computational cell, and the ds phase to contain two formula units in the computational cell.

improve the binding energy curves of both $\mathrm{H}_{2}$ and $\mathrm{LiH}^{45}$, avoiding spurious charge transfer in the dissociation limit. Spin-symmetry breaking is possible only in spin DFT, while total DFT can only get the right energies for such stretched systems with radical nonlocality. In solids, the subdivision of the lattice into spin-up and spin-down sublattices effectively doubles its size and opens a bandgap that would otherwise not be found in the spinunpolarized solution. The difficulty lower-level functionals have in describing these seemingly simple materials has garnered their title of "strongly correlated" materials. Many approximate theories outside of DFT, particularly those of Anderson ${ }^{46,47}$ within the Mott-Hubbard model Hamiltonian framework, have had success in describing the properties of strongly correlated materials.

We present PBE-only calculations of both spin-unpolarized (Unp.) and AFM $\mathrm{NiO}$ in the $\mathrm{rs}$ and Zincblende (zb) structures. Above the Néel temperature, the Unp. rs solution gives qualitatively correct ground-state spin densities; however, no gap is present in the Unp. rs DOS. The AFM rs (AF2) state is the correct zero-temperature ground state ${ }^{48}$, and PBE finds a bandgap in the DOS of approximately $0.9 \mathrm{eV}$ at equilibrium. Both gaps are severe underestimates of the fundamental gap, 3.7-4.3 $\mathrm{eV}^{49}$. (This underestimation is reduced systematically at the GGA level within the DFT $+U$ framework ${ }^{49}$.)

PBE also underestimates the equilibrium magnetic moment of rs $\mathrm{NiO}$ as $1.35 \mu_{\mathrm{B}}$ per formula unit, in contrast to experimental values in the range $1.64-1.90 \mu_{\mathrm{B}}$ per formula unit ${ }^{50}$. The oxygen ion sites acquire no magnetization. In the $z b$ phase, PBE finds the magnetic moment to be $1.51 \mu_{\mathrm{B}}$, with two formula units contributing to the magnetic moment (two Ni sites with magnetic moment 1.331 and $1.327 \mu_{\mathrm{B}}$ and two $\mathrm{O}$ sites with magnetic moment 0.341 and $0.018 \mu_{\mathrm{B}}$ ).

Reference ${ }^{49}$ suggests that the AFM zb state with antiparallel spins in bilayers along the [001] conventional cubic direction (the AF5 configuration of ref. ${ }^{48}$ ) is close in energy to the AF2-rs state near equilibrium. We have also investigated the AF5-zb state for completeness.
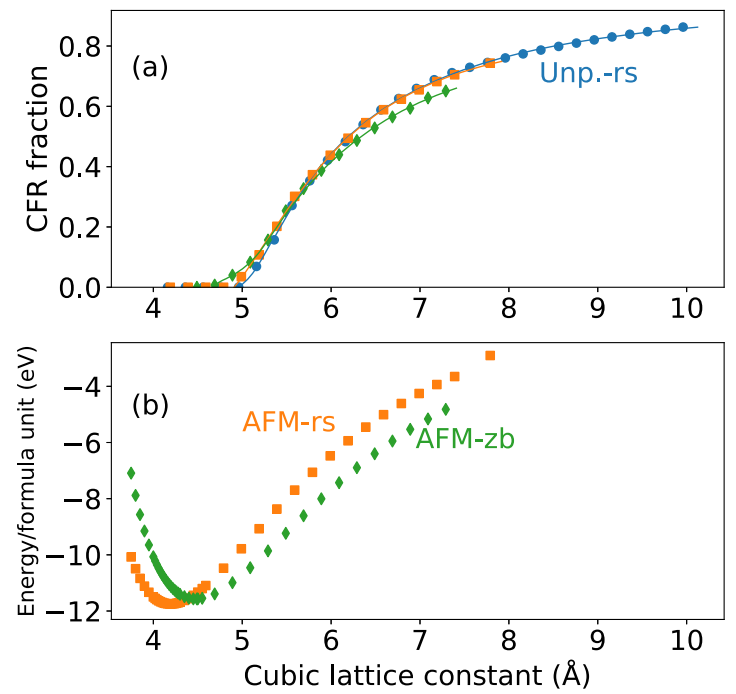

Fig. 7 CFRs of a few crystalline and magnetic phases in NiO. a Spin CFRs and $\mathbf{b}$ relative energies of AFM NiO (AF2-rs and AF5-zb) and CFR of Unp. NiO (rs) as a function of the cubic lattice constant, as calculated with PBE in VASP. The spin-up and spin-down CFRs for AFM-rs and AFM-zb NiO are identical. The Unp. rs phase of $\mathrm{NiO}$ was taken to contain one formula unit in the computational cell, the AFM rs (zb) phase to contain two (four) formula units in the computational cell.

In Fig. 7, we plot the CFRs of AFM (AF2-rs and AF5-zb) and Unp.rs NiO. The spin-up and spin-down CFRs for AFM-rs and AFM-zb $\mathrm{NiO}$ exhibit identical behavior. Moreover, the similarity of the Unp. and AFM rs curves demonstrates that the crystalline structure plays a large role in determining the size of the CFR under strain.

\section{DISCUSSION}

No bulk metal that we studied had a CFR at equilibrium, but covalent semiconductors also lack CFRs at equilibrium. Thus the fractional CFR volume at equilibrium cannot be used directly as an indicator of conductivity. CFRs emerged in all narrow gap insulators studied here (excluding C) when expanded by $40 \%$ of the equilibrium volume. Thus a semilocal calculation can recognize narrow gap insulators by either a nonzero gap (C or $\mathrm{Si})$ or an emergent CFR if $V_{\mathrm{c}} / V_{\text {eq }} \lesssim 1.4(\mathrm{Ge}$, where semilocal functionals often find zero bandgap). However, the existence of a CFR can be used as a theoretical tool in understanding the role of semilocality in describing solids.

Since standard density-gradient expansions are derived for slowly varying densities without CFRs, the absence of CFRs in metals at equilibrium, along with the short range of the exact exchange-correlation hole around an electron in a metal, suggest that the local density and its low-order derivatives suffice for an accurate approximation to the exchange-correlation energy of a metal. This argument, but based on the hole alone, was made in ref. 51 to explain why "de-orbitalization" of a meta-GGA can improve ${ }^{52}$ the magnetic moment in solid Fe.

The lack of CFRs in narrow-gap insulators suggests that semilocal functionals may still be accurate in predicting some, but not all, of their material properties. This is evident in the accuracy of LSDA and PBE in predicting the lattice geometry of Si, diamond, and the in-plane covalent bonding of graphite. However, a degree of nonlocality is needed to predict conductivity and thermodynamic properties of insulators. Weakly bonded materials like $\mathrm{Ne}$ and complex materials like $\mathrm{NiO}$ require an even higher degree of nonlocality. A truly general purpose density functional must balance the semilocality demanded by metals and 
the nonlocality needed to describe insulators, atoms, and molecules.

Just as in a small molecule, the classical turning surface at equilibrium in a periodic solid reflects the bonding type. There is no classical turning surface for typical metallic bonds and for dense networks of covalent bonds, but classical turning surfaces of characteristic shapes appear for ionic, hydrogen, and van der Waals bonds. Equation (3), with $\beta_{\mathrm{AB}}$ determined by the bond type, roughly predicts the equilibrium bond length between neighboring atoms $A$ and $B$, and might be used to find an initial guess for the geometry of a novel material. $\beta_{A B}$ falls in the same range for metallic as for covalent bonds: both show a strong overlap of the bonded atoms. CFRs in solids arise where neighboring atoms are weakly overlapped, making the local electron density relatively low; CFRs are absent when the atoms are strongly overlapped. $\beta_{\mathrm{AB}}$ $\lesssim 1$ seems to imply no CFR for solids in equilibrium with only one bond type, as shown in Table 1.

As a predictor of electrical insulation, the existence of a gap between occupied and unoccupied states in the LSDA or GGA band structure is much more reliable than the existence of a CFR. A CFR is missing not only in metals but also in many covalently bonded semiconductors and even in some AFM insulators like $\mathrm{NiO}$. The CFR in $\mathrm{NiO}$ is seemingly insensitive to the magnetic phase. For the solids studied here, our calculations found no CFRs for metals, large CFRs for wide-gap insulators, and the emergence of CFRs when small-gap semiconductors are mildly expanded.

A monovacancy in a metal can induce a CFR, and an expansive strain in any material can induce a CFR or increase its volume. In both cases, the CFR emerges in a region of relatively large density depletion, such as the interstice of stretched Si. In any solid, the fractional CFR volume (the volume of the CFR relative to a chosen cell volume) is sensitive to the crystalline phase, nearest-neighbor separation, and magnetic phase. Those wider-gap insulators with a CFR at equilibrium can be compressed until the CFR vanishes. Layered materials may have a CFR at equilibrium, at least when a density functional approximation overestimates the interlayer spacing sufficiently, as PBE does for $\mathrm{MoS}_{2}$. Our analysis of strain has been limited to homogeneous strains of cubic lattices. Applying unequal expansive strains along the high symmetry directions of the lattice would likely yield quite different CFR curves than those shown here. In materials with structures more complicated than cubic, unequal strains would likely reflect properties of the bonding along the direction of the strain, in the vein of our bonding analysis for layered materials. However, as the possibilities for this are myriad, we defer this to future study.

CFRs are also characteristic of perfect ionic and molecular crystals at equilibrium. Our analysis supports the conclusion that rare gas atoms in the crystalline phase are nearly free. Ionic crystals can have large CFRs at equilibrium (as in $\mathrm{NaCl}$ but not $\mathrm{NiO}$ ). We showed that graphite and $\mathrm{MoS}_{2}$, where intermediaterange van der Waals interactions dominate between monolayers, have CFRs located solely between monolayers and that their corresponding monolayers have no in-plane CFR. Our work demonstrates that weakly bound solids tend to have prominent CFRs. Hydrogen-bonded crystals like ice, while not tested here, can be expected to have substantial CFR volume fractions, as suggested by Fig. 8 for the water dimer in ref. ${ }^{2}$.

\section{METHODS \\ Computational details}

All calculations were performed with either the VASP ${ }^{53-56}$, or the Castep $\operatorname{code}^{57,58}$, or both. All GGA calculations used the PBE GGA ${ }^{3}$, and all LSDA calculations used the Perdew-Zunger parameterization of the uniform electron gas correlation energy ${ }^{59}$. The calculations in VASP were performed with a cutoff energy of $800 \mathrm{eV}$, a 「-centered mesh of spacing $0.08 \AA^{-1}$, energy convergence of $10^{-6} \mathrm{eV}$, and stress convergence at $10^{-3} \mathrm{eV} / \AA \AA$. To determine equilibrium geometries in VASP, for metals, first-order
Methfessel-Paxton smearing with parameter of 0.2 was used, and for insulators, the Blöchl tetrahedron method was used. VASP's internal methods were used to determine the relaxed cell volume.

All calculations were spin-unpolarized, except those for AFM NiO. For AFM NiO, the relaxed volume was determined by fitting to the SJEOS ${ }^{44}$. Convergence was aided using a linear magnetization-density mixing scheme $\left(A M I X=0.2\right.$, AMIX_MAG $\left.=0.8, B M I X=B M I X \_M A G=0.0001\right)$. From ref. ${ }^{48}$, the ground state of AFM NiO has antiparallel spins along the [111] direction of the conventional cubic cell (AF2 ordering, a four-ion basis), which was used here. Magnetic initialization of $\pm 2 \mu_{\mathrm{B}}$ was given for the $\mathrm{Ni}$ atoms and $0 \mu_{\mathrm{B}}$ for the $\mathrm{O}$ atoms.

Approximate bandgaps were calculated from the DOS, on the default resolution for VASP (300 total sampling points). Increasing the resolution of the DOS to 3000 sampling points gave changes at most of $0.05 \mathrm{eV}$ only near equilibrium for $\mathrm{Si}$. For $\mathrm{Ge}$, no change in the DOS gap could be discerned.

In Castep, a density-mixing algorithm was used to reach self-consistency, and geometries were determined with a BFGS (Broyden-Fletcher-Goldfarb-Shanno) energy minimization scheme with the finite basis set corrected for stress ${ }^{60}$. After relaxation, a calculation at the equilibrium volume using the Blöchl tetrahedron method was performed to accurately determine the DOS. Accurate ${ }^{61}$ PAW on-the-fly pseudopotentials were used throughout. Supplementary Tables VIII-LIII present all raw data.

For monolayers, a $45 \times 45 \times 1 \mathbf{k}$-point grid was used in conjunction with the Blöchl tetrahedron method. All other parameters remain the same from bulk calculations. The $c$ direction was padded with $30 \AA$ of vacuum region to reduce interactions between image monolayers.

In density functional plane-wave codes, the densities and potentials are stored on a uniform grid $\mathbf{R}$, the dimensions of which are determined by the size of the UC and the plane-wave cutoff energy. Acceptable convergence of the total energy relies on suitable convergence of the potentials and densities on this grid. The values of $v_{s}(\mathbf{R})$ are obtained from this grid. In core regions, the true potential is much deeper than the pseudopotential, so these are classically allowed. Thus the PAW pseudopotential core regions were excluded from the CFR. (Frozen-core pseudopotentials were used in both VASP and Castep.)

The self-consistent electronic eigenstates give $\varepsilon_{\mathrm{HO}}$ (the Fermi energy $\varepsilon_{\mathrm{F}}$ in a metal), and the regions where $\varepsilon_{\mathrm{HO}}-v_{\mathrm{s}}(\mathbf{R})<0$ define the CFR. We assign equal volume to each point relative to the $U C$, as the real-space mesh is uniform. To find the volume of a CFR relative to a given cell volume, we need to average over a UC or repeat-unit of the periodic crystal. But such a UC must in some cases contain more than one atom. For ease of comparison, we define and report $V_{\mathrm{UC}}$ as the volume per atom of the UC, which for a given material is of course independent of the choice of UC (primitive, conventional, etc.). Similarly, we define $N_{U C}$ as the number of mesh points per atom of the UC. Then the volume of any mesh point is $V_{\mathrm{UC}} / N_{\mathrm{UC}}$. If there are $N_{\mathrm{CFR}}$ points at which $\varepsilon_{\mathrm{HO}}-v_{\mathrm{s}}(\mathbf{r})<0$, the volume per atom of the CFR is

$V_{\mathrm{CFR}}=V_{\mathrm{UC}} N_{\mathrm{CFR}} / N_{\mathrm{UC}}$.

The dimensionless "fractional volume" of the CFR, which was used throughout, is defined as

$v \equiv V_{\mathrm{CFR}} / V_{\mathrm{UC}}=N_{\mathrm{CFR}} / N_{\mathrm{UC}}$,

the number of real-space mesh points within the CFR relative to the total number of mesh points in the UC.

As the fractional CFR volume $v \rightarrow 0$, our method requires ever finer realand reciprocal-space meshes to resolve $v$. This need is limited by the resolution determined by the plane-wave cutoff energy. Our data for $v \ll 1$ will necessarily be more noisy than for larger values of $v$. Despite this, we show a posteriori that reasonable fits to $v\left(V_{U C}\right)$ may be found.

Each code uses differently generated pseudopotentials with different optimal basis set cutoff energies (and hence pseudopotential grid sizes, etc.), different energy minimization schemes, and different Brillouin zone integration methods. To ensure that our method is not dependent upon the numerical methods of a particular code, we have verified that the Castep and VASP results are consistent.

\section{DATA AVAILABILITY}

All data supporting the findings of this work are available in the article and its Supplementary Information. Extra data and machine readable data are publicly available at the Materials Cloud Archive, 10.24435/materialscloud:2h-zq ${ }^{68}$. 


\section{CODE AVAILABILITY}

The Python3 code used to analyze the VASP outputs is publicly available at https:// gitlab.com/dhamil/vasp-utilities. Access to the repository is unrestricted; any queries should be directed to the corresponding author.

Received: 3 July 2020; Accepted: 12 December 2020; Published online: 01 February 2021

\section{REFERENCES}

1. Kohn, W. \& Sham, L. J. Self-consistent equations including exchange and correlation effects. Phys. Rev. 140, A1133-A1138 (1965).

2. Ospadov, E., Tao, J., Staroverov, V. N. \& Perdew, J. P. Visualizing atomic sizes and molecular shapes with the classical turning surface of the Kohn-Sham potential. Proc. Natl Acad. Sci. USA 115, E11578-E11585 (2018).

3. Perdew, J. P., Burke, K. \& Ernzerhof, M. Generalized gradient approximation made simple. Phys. Rev. Lett. 77, 3865-3868 (1996).

4. Burke, K., Perdew, J. P. \& Ernzerhof, M. Why semilocal functionals work: accuracy of the on-top pair density and importance of system averaging. J. Chem. Phys. 109, 3760-3771 (1998)

5. Perdew, J. P., Ernzerhof, M., Burke, K. \& Savin, A. On-top pair-density interpretation of spin density functional theory, with applications to magnetism. Int. J. Quantum Chem. 61, 197-205 (1997).

6. Ekholm, M. et al. Assessing the SCAN functional for itinerant electron ferromagnets. Phys. Rev. B 98, 094413 (2018)

7. Fu, Y. \& Singh, D. J. Density functional methods for the magnetism of transition metals: SCAN in relation to other functionals. Phys. Rev. B 100, 045126 (2019).

8. Sun, J., Perdew, J. P. \& Ruzsinszky, A. Semilocal density functional obeying a strongly tightened bound for exchange. Proc. Natl Acad. Sci. USA 112, 685-689 (2015).

9. Sun, J., Ruzsinszky, A. \& Perdew, J. P. Strongly constrained and appropriately normed semilocal density functional. Phys. Rev. Lett. 115, 036402 (2015).

10. Heyd, J., Scuseria, G. E. \& Ernzerhof, M. Hybrid functionals based on a screened Coulomb potential. J. Chem. Phys. 118, 8207-8215 (2003).

11. Shahi, C., Sun, J. \& Perdew, J. P. Accurate critical pressures for structural phase transitions of group IV, III-V, and II-VI compounds from the SCAN density functional. Phys. Rev. B 97, 094111 (2018).

12. Zhang, Y. et al. Efficient first-principles prediction of solid stability: towards chemical accuracy. npj Comput. Mater. 4, 9 (2018).

13. Skone, J. H., Govoni, M. \& Galli, G. Self-consistent hybrid functional for condensed systems. Phys. Rev. B 89, 195112 (2014).

14. Mott, N. F. \& Fowler, R. H. The electrical conductivity of transition metals. Proc. $R$. Soc. A 153, 699-717 (1936).

15. Kohn, W. Theory of the insulating state. Phys. Rev. 133, A171-A181 (1964).

16. Kohn, W. In Metals and Insulators 353-411 (Gordon and Breach, New York, 1967).

17. Ashcroft, N. W. \& Mermin, N. D. Solid State Physics (Holt, Rinehart and Winston, 1976)

18. Perdew, J. P., Parr, R. G., Levy, M. \& Balduz Jr., J. L. Density-functional theory for fractional particle number: derivative discontinuities of the energy. Phys. Rev. Lett. 49, 1691-1694 (1982).

19. Engel, E. \& Dreizler, R. M. From explicit to implicit density functionals. J. Comput. Chem. 20, 31-50 (1999).

20. Ospadov, E., Ryabinkin, I. G. \& Staroverov, V. N. Improved method for generating exchange-correlation potentials from electronic wave functions. J. Chem. Phys. 146, 084103 (2017)

21. Yang, Z.-Z. \& Davidson, E. R. Evaluation of a characteristic atomic radius by an ab initio method. Int. J. Quantum Chem. 62, 47-53 (1997).

22. Yang, Z.-Z. \& Zhao, D.-X. A characteristic molecular contour evaluated by a theoretical method. Chem. Phys. Lett. 292, 387-393 (1998).

23. Burke, K., Cancio, A., Gould, T. \& Pittalis, S. Locality of correlation in density functional theory. J. Chem. Phys. 145, 054112 (2016).

24. Gould, T., Liberles, B. T. \& Perdew, J. P. What do we learn from the classical turning surface of the Kohn-Sham potential as electron number is varied continuously? J. Chem. Phys. 152, 054105 (2020).

25. Elliott, P., Lee, D., Cangi, A. \& Burke, K. Semiclassical origins of density functionals. Phys. Rev. Lett. 100, 256406 (2008).

26. Kohn, W. \& Sham, L. J. Quantum density oscillations in an inhomogeneous electron gas. Phys. Rev. 137, A1697-A1705 (1965).

27. Perdew, J. P. In Density Functional Methods In Physics 265-308 (Plenum, New York, 1985).

28. Perdew, J. P. Density functional theory and the band gap problem. Int. J. Quantum Chem. Symp. 19, 497-523 (1986)

29. Grüning, M., Marini, A. \& Rubio, A. Density functionals from many-body perturbation theory: the band gap for semiconductors and insulators. J. Chem. Phys. 124, 154108 (2006)
30. Perdew, J. P. et al. Understanding band gaps of solids in generalized Kohn-Sham theory. Proc. Natl Acad. Sci. USA 114, 2801-2806 (2017).

31. van Leeuwen, R., Gritsenko, O. \& Baerends, E. J. Step structure in the atomic Kohn-Sham potential. Z. Phys. D 33, 229-238 (1995).

32. Hodgson, M. J. P., Kraisler, E., Schild, A. \& Gross, E. K. U. How interatomic steps in the exact Kohn-Sham potential relate to derivative discontinuities of the energy. J. Phys. Chem. Lett. 8, 5974-5980 (2017).

33. Becke, A. D. \& Edgecombe, K. E. A simple measure of electron localization in atomic and molecular systems. J. Chem. Phys. 92, 5397-5403 (1990).

34. Pham, C. H., Reddy, S. K., Chen, K., Knight, C. \& Paesani, F. Many-body interactions in ice. J. Chem. Theory Comput. 13, 1778-1784 (2017).

35. Harrison, W. A. Pseudopotentials in the Theory of Metals (Benjamin, New York, 1966).

36. Schwerdtfeger, P. \& Hermann, A. Equation of state for solid neon from quantum theory. Phys. Rev. B 80, 064106 (2009).

37. He, Y.-g., Tang, X.-z. \& Pu, Y.-k First-principle study of solid neon under high compression. Physica B 405, 4335-4338 (2010).

38. Ma, Y. et al. Transparent dense sodium. Nature 458, 182-185 (2009).

39. Perdew, J. P., Wang, Y. \& Engel, E. Liquid-drop model for crystalline metals: vacancy-formation, cohesive, and face-dependent surface energies. Phys. Rev. Lett. 66, 508-511 (1991).

40. Carling, K. et al. Vacancies in metals: From first principles calculations to experimental data. Phys. Rev. Lett. 85, 3862-3865 (2000).

41. Constantin, L. A., Pitarke, J. M., Dobson, J. F., Garcia-Lekue, A. \& Perdew, J. P. High-level correlated approach to the jellium surface energy without uniformgas input. Phys. Rev. Lett. 100, 036401 (2008).

42. Constantin, L. A., Snyder, J. C., Perdew, J. P. \& Burke, K. Communication: Ionization potentials in the limit of large atomic number. J. Chem. Phys. 133, 241103 (2010).

43. Perdew, J. P., Ruzsinszky, A., Sun, J., Nepal, N. K. \& Kaplan, A. D. Interpretations of ground-state symmetry breaking and strong correlation in wavefunction and density functional theories. PNAS 118, https://doi.org/10.1073/pnas.2017850118. In press. (2021).

44. Staroverov, V. N., Scuseria, G. E., Tao, J. \& Perdew, J. P. Tests of a ladder of density functionals for bulk solids and surfaces. Phys. Rev. B 69, 075102 (2004).

45. Fuks, J. I., Rubio, A. \& Maitra, N. T. Charge transfer in time-dependent densityfunctional theory via spin-symmetry breaking. Phys. Rev. A 83, 042501 (2011).

46. Anderson, P. W. An approximate quantum theory of the antiferromagnetic ground state. Phys. Rev. 86, 694-701 (1952).

47. Anderson, P. W. Localized magnetic states in metals. Phys. Rev. 124, 41-53 (1961).

48. Schrön, A., Rödl, C. \& Bechstedt, F. Energetic stability and magnetic properties of $\mathrm{MnO}$ in the rocksalt, wurtzite, and zinc-blende structures: influence of exchange and correlation. Phys. Rev. B 82, 165109 (2010).

49. Peng, H. \& Perdew, J. P. Synergy of van der Waals and self-interaction corrections in transition metal monoxides. Phys. Rev. B 96, 100101 (2017).

50. Tran, F., Blaha, P., Schwarz, K. \& Novák, P. Hybrid exchange-correlation energy functionals for strongly correlated electrons: applications to transition-metal monoxides. Phys. Rev. B 74, 1-10 (2006)

51. Furness, J. W., Kaplan, A. D., Ning, J., Perdew, J. P. \& Sun, J. Accurate and numerically efficient $r^{2}$ SCAN meta-generalized gradient approximation. J. Phys. Chem. Lett. 11, 8208-8215 (2020).

52. Mejía-Rodríguez, D. \& Trickey, S. B. Meta-GGA performance in solids at almost GGA cost. Phys. Rev. B 102, 121109 (2020).

53. Kresse, G. \& Hafner, J. Ab initio molecular dynamics for liquid metals. Phys. Rev. $B$ 47, 558-561 (1993)

54. Kresse, G. \& Hafner, J. Ab initio molecular-dynamics simulation of the liquidmetalamorphous-semiconductor transition in germanium. Phys. Rev. B 49, 14251-14269 (1994).

55. Kresse, G. \& Furthmüller, J. Efficient iterative schemes for ab initio total-energy calculations using a plane-wave basis set. Phys. Rev. B 54, 11169-11186 (1996).

56. Kresse, G. \& Furthmüller, J. Efficiency of ab-initio total energy calculations for metals and semiconductors using a plane-wave basis set. Comput. Mater. Sci. 6 , 15-50 (1996).

57. Segall, M. D. et al. First-principles simulation: ideas, illustrations and the CASTEP code. J. Phys. Condens. Matter 14, 2717-2744 (2002).

58. Clark, S. J. et al. First principles methods using CASTEP. Z. Kryst. 220, 567-570 (2005)

59. Perdew, J. P. \& Zunger, A. Self-interaction correction to density-functional approximations for many-electron systems. Phys. Rev. B 23, 5048-5079 (1981).

60. Francis, G. P. \& Payne, M. C. Finite basis set corrections to total energy pseudopotential calculations. J. Phys. Condens. Matter 2, 4395-4404 (1990).

61. Lejaeghere, K. et al. Reproducibility in density functional theory calculations of solids. Science 351, 6280 (2016).

62. Böker, T. et al. Band structure of $\mathrm{MoS}_{2}, \mathrm{MoSe}_{2}$, and $a-\mathrm{MoTe}_{2}$ : angle-resolved photoelectron spectroscopy and ab initio calculations. Phys. Rev. B 64, 23505 (2001).

63. Kan, M. et al. Structures and phase transition of a $\mathrm{MoS}_{2}$ monolayer. J. Phys. Chem 118, 1515-1522 (2014) 
64. Sun, J. et al. Self-consistent meta-generalized gradient approximation within the projector-augmented-wave method. Phys. Rev. B 84, 035117 (2011).

65. Haas, P., Tran, F. \& Blaha, P. Calculation of the lattice constant of solids with semilocal functionals. Phys. Rev. B 79, 085104 (2009).

66. Zhao, Y. X. \& Spain, I. L. X-ray diffraction data for graphite to $20 \mathrm{GPa}$. Phys. Rev. B 40, 993-997 (1989).

67. Trucano, P. \& Chen, R. Structure of graphite by neutron diffraction. Nature $\mathbf{2 5 8}$, 136-137 (1975)

68. Kaplan, A., Clark, S., Burke, K. \& Perdew, J. Calculation and interpretation of classical turning surfaces in solids. Materials Cloud Archive 2020.169, https://doi. org/10.24435/materialscloud:2h-zq (2020).

\section{ACKNOWLEDGEMENTS}

A.D.K. acknowledges the support of the Department of Energy (DOE), Basic Energy Sciences under grant No. DE-SC0012575 and support from Temple University. S.J.C. acknowledges Engineering and Physical Sciences Research Council support on grant EP/P022782/1. K.B. was supported by DOE under grant no. DE-FG02-08ER46496. J.P.P. acknowledges the support of the National Science Foundation under grant number DMR-1939528.

\section{AUTHOR CONTRIBUTIONS}

A.D.K. and S.J.C. performed the calculations and analyzed the data. K.B. and J.P.P. designed the research. All authors contributed to designing the research methods, interpreting the data, and writing the manuscript.

\section{COMPETING INTERESTS}

The authors declare no competing interests.

\section{ADDITIONAL INFORMATION}

Supplementary information is available for this paper at https://doi.org/10.1038/ s41524-020-00479-0.

Correspondence and requests for materials should be addressed to S.J.C., K.B. or J.P.P.

Reprints and permission information is available at http://www.nature.com/ reprints

Publisher's note Springer Nature remains neutral with regard to jurisdictional claims in published maps and institutional affiliations.
Open Access This article is licensed under a Creative Commons Attribution 4.0 International License, which permits use, sharing, adaptation, distribution and reproduction in any medium or format, as long as you give appropriate credit to the original author(s) and the source, provide a link to the Creative Commons license, and indicate if changes were made. The images or other third party material in this article are included in the article's Creative Commons license, unless indicated otherwise in a credit line to the material. If material is not included in the article's Creative Commons license and your intended use is not permitted by statutory regulation or exceeds the permitted use, you will need to obtain permission directly from the copyright holder. To view a copy of this license, visit http://creativecommons. org/licenses/by/4.0/.

(c) The Author(s) 2021 